\title{
Ubiquitin Immunochemistry as a Diagnostic Aid for Community Pathologists Evaluating Patients Who Have Dementia
}

Charleen T. Chu, M.D., Ph.D., James L. Caruso, M.D., Thomas J. Cummings, M.D., John Ervin, B.A., Carlyn Rosenberg, M.S., Christine M. Hulette, M.D.

Department of Pathology, Division of Neuropathology, University of Pittsburgh Medical Center (CTC), Pittsburgh, Pennsylvania; Department of Pathology, U.S. Naval Hospital (JLC), Pensacola, Florida; Departments of Pathology (TJC, CMH), Medicine (CMH), and Anesthesiology (JLC), Duke University Medical Center, and Bryan Alzheimer's Disease Research Center (JE, CR, CMH), Durham, North Carolina

\begin{abstract}
Alzheimer's disease is the most common cause of dementia. It is associated with genetic risk factors and at least three autosomal dominant mutations. Community pathologists are frequently asked by families to evaluate autopsy material for Alzheimer's disease. Neuropathologic diagnosis is based on technically difficult silver impregnation stains that may not be readily available to communitybased pathologists. Because immunohistochemical techniques are more widely accessible, we evaluated the practical utility of using a single immunohistochemical stain for diagnosing Alzheimer's disease. The ubiquitin antigen was selected because of its presence in morphologically distinct deposits characteristic of several neurodegenerative diseases. Paraffin blocks were obtained from the Bryan Alzheimer's Disease Research Center Brain Bank, a repository of approximately 900 brains. Tissues from 16 individuals who exhibited the entire range of Alzheimer's-type neuropathology were selected. Ubiquitin immunostains, evaluated blindly and independently by four pathologists ranging from firstyear resident trainee to experienced neuropathologist, reliably stained both neuritic plaques and neurofibrillary tangles essential for diagnosing and staging Alzheimer's disease. Nondemented controls with early Alzheimer's-type changes were easily distinguished from cases of definitive Alzheimer's disease. The stains also highlighted characteristic inclusions of Parkinson's disease or Lewy body dementia. Ubiquitin immunohistochemistry is a reliable, reproducible, and readily available diagnos-
\end{abstract}

Copyright () 2000 by The United States and Canadian Academy of Pathology, Inc.

VOL. 13, NO. 4, P. 420, 2000 Printed in the U.S.A

Date of acceptance: September 23, 1999.

Address reprint requests to: Christine M. Hulette, M.D., Department of Pathology, Neuropathology Section, Box 2900, Duke University Medical Center, Durham, NC 27710; fax: 919-684-6514. tic aid for distinguishing Alzheimer's disease from other causes of dementia.

KEY WORDS: Alzheimer's disease, Dementia, Immunohistochemistry, Parkinson's disease, Ubiquitin.

Mod Pathol 2000;13(4):420-426

Alzheimer's disease is the most common form of dementia. Because of its genetic predispositions, the definitive diagnosis of Alzheimer's disease is important to families. Mutations in the genes encoding the presenilins and $\beta$-amyloid precursor protein have been identified in several families, and an early-onset form is associated with trisomy 21 ( 1 , 2 ). Moreover, several genetic risk factors, such as the apolipoprotein E4 allele and other ligands of the $\alpha_{2}$-macroglobulin receptor (3), have been identified $(4,5)$. Although genetic risk factors are helpful in the clinical disease management of patients who have dementia (6), the definitive diagnosis of Alzheimer's disease rests on histopathologic examination of the brain postmortem.

Most neuropathologists use technically difficult silver impregnation stains to identify the neuritic plaques and neurofibrillary tangles that are characteristic of Alzheimer's disease (7). Guidelines developed by the Consortium to Establish a Registry for Alzheimer's Disease (CERAD) involve the histopathologic examination of specific brain regions using silver impregnation (e.g., Bielschowsky) stains to highlight neuritic plaques and tangles. CERAD diagnosis of definite, probable, and possible Alzheimer's disease relies on semiquantitative assessment of plaque density in the neocortex combined with clinical information (7). In a multi-institutional study involving 24 neuropathologists, rapid semiquantitative assessments of plaque density obtained by scanning low power fields for regions of maximum involvement showed greater inter-rater 
reliability than attempts to count plaque numbers per square millimeter field (8). Although tangle density is not formally used in this diagnostic scheme, tangles in specific regions of the temporal and occipital lobes are important for the Braak neuropathologic staging system that correlates with disease duration (9). Information from both CERAD and Braak analyses was incorporated into a revised set of criteria for classifying dementia brains (10). The need for increased neuropathologic standardization in dementia research has promoted a growing armamentarium of special stains that are routinely used in large academic centers to assess brains from patients who had dementia.

For the practicing pathologist with more limited laboratory resources, simplifying the diagnostic evaluation of dementia brains is desirable. Ubiquitin, a small protein tag that marks damaged proteins that are destined for degradation by proteasomes (11), accumulates in both plaques and tangles. In addition, ubiquitin is present in Lewy bodies, a pathologic inclusion that is characteristic of Parkinson's disease. Identification of Lewy bodies in patients who are suspected of having Alzheimer's disease is important, as more than $20 \%$ of pathologically confirmed cases of Alzheimer's disease exhibit concurrent Parkinson's disease pathology $(12,13)$. Moreover, in the absence of Alzheimer's-type changes, diffuse Lewy body pathology itself is an alternative cause of dementia. Considering the ease of our chosen immunohistochemistry technique and its wide availability, we evaluated the utility of ubiquitin immunostaining for the neuropathologic diagnosis of patients who are suspected of having Alzheimer's disease. We found that this single immunohistochemical study evaluated independently of other histologic studies allowed both general pathologists-in-training and neuropathologists to render accurate, reproducible diagnoses.

\section{MATERIALS AND METHODS}

\section{Pathologic Material}

Paraffin blocks were obtained from the Bryan Alzheimer's Disease Research Center Brain Bank, a repository of approximately 900 brains (14). All diagnoses in the bank were rendered by an experienced neuropathologist $(\mathrm{CMH})$ based on examination of hematoxylin and eosin (H\&E) stains, microwave King silver impregnation stains (15), and Congo red amyloid stains. Tissues from 16 individuals who exhibited the entire range of Alzheimer's-type neuropathology were selected randomly from each diagnostic category by an individual not involved in analysis (CR).

\section{Ubiquitin Immunohistochemistry}

The sections were cut $8 \mu$ thick from paraffinembedded tissue blocks and mounted directly onto Superfrost/Plus slides (Fisher Scientific, Pittsburgh, $\mathrm{PA})$. The slides were baked in a $37^{\circ} \mathrm{C}$ incubator for $12 \mathrm{~h}$ before deparaffinization. After the slides were washed in phosphate-buffered saline (PBS) pH 7.6, they were pretreated with endogenous peroxide blocker Endo/Blocker (Biomeda, Foster City, CA). After a thorough PBS washing and wiping excess buffer away from the tissue, a prediluted rabbit polyclonal antibody recognizing human ubiquitin was applied with a dropper to the tissue. The primary antibody incubated for $30 \mathrm{~min}$ at $37^{\circ} \mathrm{C}$ in an incubation chamber before washing in PBS. After blotting away excess PBS, a prediluted biotinylated $2^{\circ}$ was applied with a dropper and incubated for 20 min at $37^{\circ} \mathrm{C}$. After another PBS wash, a prediluted streptavidin conjugated horseradish peroxidase was applied with a dropper and incubated for 20 min at $37^{\circ} \mathrm{C}$. Both prediluted reagents are contained in the Autoprobe III Reagent Sets (Biomeda, Foster City, CA). Peroxide/diaminobenzidine,3,3'diaminobenzidine (Sigma, St. Louis, MO) was used as the chromogen. Nuclei were counterstained with Biomeda's aqueous hematoxylin applied with a dropper for 20 seconds before washing off the excess in tap water to avoid overstaining. Slides were blued in ammonical water, then washed in tap water before dehydrating and coverslipping. For negative control sections, nonimmune serum was used in place of the primary polyclonal antibody.

\section{Scoring of Immunostains}

The ubiquitin immunostains were reviewed independently by four pathologists using the indicated template (Fig. 1). (For more details on selection of appropriate brain sections during gross examination and assessing these regions by CERAD and Braak criteria, see Mirra et al. (7) Fig. 1 and Braak and Braak (9) Fig. 14.) The reviewers-an experienced neuropathologist (CMH), a neuropathology fellow (CTC), a senior anatomic pathology/clinical pathology resident (JLC), and a first-year anatomic pathology resident with brief exposure to autopsy neuropathology (TJC)—were blinded to age, history, H\&E histology, and original diagnoses during the semiquantitative scoring of these areas (Fig. 1, top). The cases were then categorized with respect to CERAD criteria by factoring in age and history of dementia, and the Braak stage was assessed using a simplified algorithm (Fig. 1, bottom). Other pathologic features, such as Lewy bodies or vascular changes, were noted. After 4 to $8 \mathrm{mo}$, the slides were scored blindly a second time by the senior reviewers. 


\begin{tabular}{|l|c|c|c|}
\hline & $\emptyset /$ sparse/moderate/frequent & Ø/modest/numerous & Y/N \\
\hline Section & PLAQUES & TANGLES & Lewy bodies \\
\hline MF cortex & & & \\
\hline IP cortex & & & \\
\hline ST cortex & & & \\
\hline entorhinal cortex & & & \\
\hline CAl hippocampus & & & \\
\hline temporal isocortex & & & \\
\hline $1^{\circ}$ visual cortex & & & \\
\hline
\end{tabular}

\begin{tabular}{|l|l|}
\hline substantia nigra & cell loss? \\
\hline vascular disease (describe): & Lewy bodies? \\
\hline \hline AGE & History of dementia?
\end{tabular}

CERAD Plaque score (Most severely affected cortex section) (circle one) Frequent $=\mathbf{C} \quad$ Moderate $=\mathbf{B}$ if $>75$ yrs old $\quad$ Sparse $=\mathbf{A}$ if $>75$ C if $<$ or $=75 \quad$ B if $50-75$

CERAD Diagnosis

Plaque score $B$ with history of dementia

Plaque score A with history of dementia Possible AD

Plaque score $\mathrm{B} / \mathrm{C}$ with no dementia Possible $\mathrm{AD}$

All others

Non-AD

Braak Stage: I II III IV V VI (circle one)

Transentorhinal: Modest (I) to numerous (II) tangles limited to entorhinal, transentorhinal cortex.

Limbic: Plus Modest (III) to numerous (IV) tangles elsewhere in temporal lobe Neocortical: Plus Modest (V) to numerous (VI) tangles in primary visual cortex

Parkinson's Cortical Lewy bodies Vascular disease (circle if applicable)

FIGURE 1. Worksheet used for evaluating dementia brains using ubiquitin immunostains. Top, semiquantitative assessments of plaque density in three cortical sections taken according to the protocol of the Consortium to Establish a Registry for Alzheimer's Disease and of tangle density in sections for Braak staging were recorded from ubiquitin immunostained sections. The presence or absence of Lewy bodies, nigral neuron loss, nigral tangles, vascular abnormalities, lacunes, or infarcts were noted. At this time, reviewers were blinded to age and clinical history. Bottom, Alzheimer's disease and Parkinson's/ Lewy body diseases were diagnosed according to criteria of the Consortium to Establish a Registry for Alzheimer's Disease. Our simplification of the Braak staging scheme is outlined on the worksheet.

\section{Data Analysis}

For the purposes of calculating sensitivity, specificity, and predictive values of ubiquitin immunostain scoring, each review of each case was counted as a separate test. For example, if two of the four reviewers scored the 16 cases twice, the total number of tests performed was $64(4 \times 16)$. For diagnosis by CERAD criteria, probable or definite Alzheimer's disease was considered a positive result. If a reviewer scored the case as possible-probable, then the test was considered 0.5 positive and 0.5 negative. Likewise, if a reviewer questioned the possible presence of Lewy bodies, then this was counted as 0.5 positive and 0.5 negative. Specificity was calculated as true negative/(true negative + false positive), sensitivity as true positive/(true positive + false negative), positive predictive value as true positive/(true positive + false positive), and negative predictive value as true negative/ (true negative + false negative).

\section{RESULTS}

Plaques and tangles were readily visualized on ubiquitin-stained sections (Fig. 2). The subjective impression of all four reviewers was that the immunostains were more easily interpreted than were silver impregnation stains because the background of unaffected brain is cleaner and the counterstain highlights cytoarchitectural features (Fig. 3). Moreover, accurate classification of the cases into low and high probability categories for Alzheimer's disease was achieved by each of the reviewers, independent of experience level (Table 1). The sensitivity was $92.5 \%$, specificity was $97 \%$, positive predictive value was $98 \%$, and negative predictive value was $89 \%$.

Ubiquitin immunostains also allowed fairly accurate assessments of tangle density in different regions of the brain. However, the success rate of using the stain for Braak staging was more variable (Table 2). Experience seemed to be a factor, particularly the ability to identify cytoarchitectural features that define the entorhinal cortex or primary visual cortex. Correct categorization into transentorhinal (I-II), limbic (III-IV), or neocortical (V-VI) stages occurred only 56 to $73 \%$ of the time, although $86 \%$ of scores were within one stage $( \pm)$ of the official silver stain stage. In certain regions, such as the primary visual cortex, ubiquitin stains seemed to be more sensitive in detecting tangles (Fig. 3).

Cortical Lewy bodies can be difficult to discern by routine histology $(10,13,16)$, but both cortical and nigral Lewy bodies were readily identified by ubiquitin immunostaining (Fig. 4). Cases 6, 8, 10, and 11 had pathologic diagnoses of Parkinson's disease and/or diffuse Lewy body disease in combination with varying stages of Alzheimer's-type changes. Ubiquitin immunostains allowed identification of these cases with a sensitivity of $83 \%$ and a negative predictive value of $92.5 \%$. For Case 7 , all reviewers noted the definite presence of Lewy bodies by ubiquitin immunostaining. Re-review of the original $\mathrm{H} \& \mathrm{E}$ stain revealed cortical Lewy bodies that had been missed. After correcting the official diagnosis for Case 7, the specificity and positive predictive values of ubiquitin stains for identifying Lewy bodies were $95 \%$ and $88 \%$, respectively. Increased exposure to neuropathology (Reviewers 1 and 2) was correlated with greater diagnostic accuracy (sensitivity of $99 \%$, specificity of $90 \%$, and predictive values of 96 to $97 \%$ ).

Although the blood vessels stained intensely for ubiquitin in several cases, this did not correlate with the presence of amyloid as assessed by Congo red stains. 

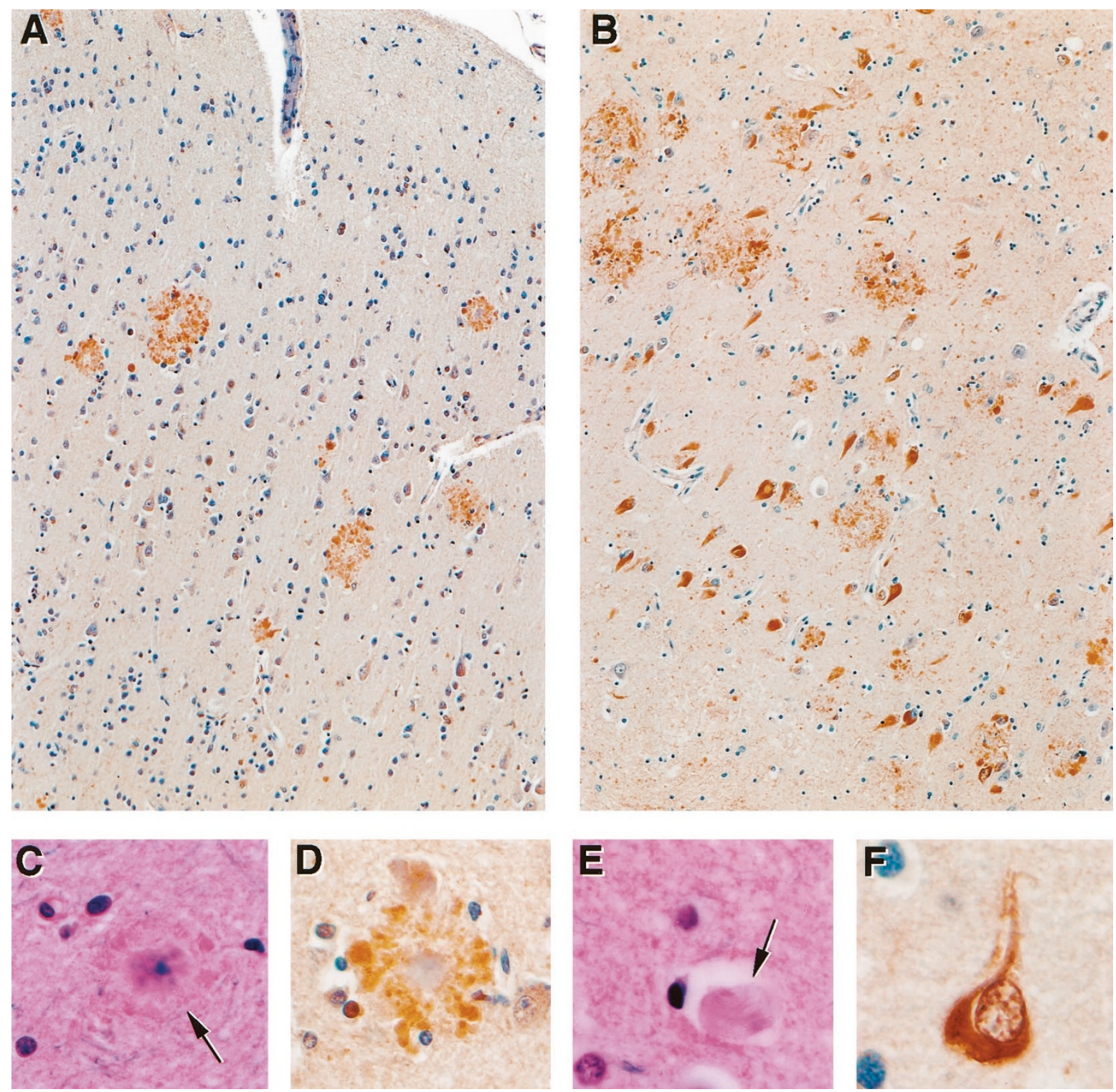

FIGURE 2. Plaques and tangles by ubiquitin immunochemistry. A, cortical section with moderate density of neuritic plaques. Note sharp contrast with the hematoxylin-stained background, which clearly delineates cytoarchitectural and vascular patterns (Case 5 , original magnification, $33 \times$, ubiquitin). B, numerous plaques and tangles from the hippocampal formation. Because tangles conform to the shape of the neuron, flame-shaped tangles are prevalent in this region (Case 11, original magnification, $33 \times$, ubiquitin). C, typical mature neuritic plaque (arrow) stained by Luxol fast blue (LFB)/hematoxylin and eosin (H\&E). Plaques are even more subtle without the LFB stain because the amyloid core stains pink on H\&E (original magnification, $132 \times$ ). D, a mature neuritic plaque stained by anti-ubiquitin is much easier to discern (Case 5 , original magnification, $100 \times$ ). E, tangles (arrow) are difficult to discern without special stains (original magnification, 198 $\times$, LFB/H\&E). F, note the fibrillar appearance of the ubiquitin-stained tangle, which conforms to the shape of the cell, filling the cytoplasm and wrapping around the nucleus. See also Figure 4D for a tangle in a round neuron (Case 8 , original magnification, $200 \times$ ).

\section{DISCUSSION}

In this study, we evaluated the usefulness of ubiquitin immunostains for neuropathologic confirmation of Alzheimer's disease in a series of patients who exhibited the entire range of age- or disease-related Alzheimer's-type neuropathologic changes. In particular, we were interested in determining whether this single immunostain would be useful for community pathologists who do not have extensive training in neuropathology and/or immunohistochemistry. We recognize that some immunohistochemistry can be technically demanding.
However, because of the convenience of Biomeda's prediluted products, this avidin biotin complex system was used for its easy application. After the staining results for this study were evaluated, it was concluded that the level of signal obtained was consistent and reproducible. Nondemented age controls with mild Alzheimer's-type changes were easily distinguished from cases of probable or definitive Alzheimer's disease. The stains also highlighted Lewy bodies characteristic of Parkinson's disease, diffuse Lewy body dementia, or Alzheimer's disease with Lewy bodies. If one were to 

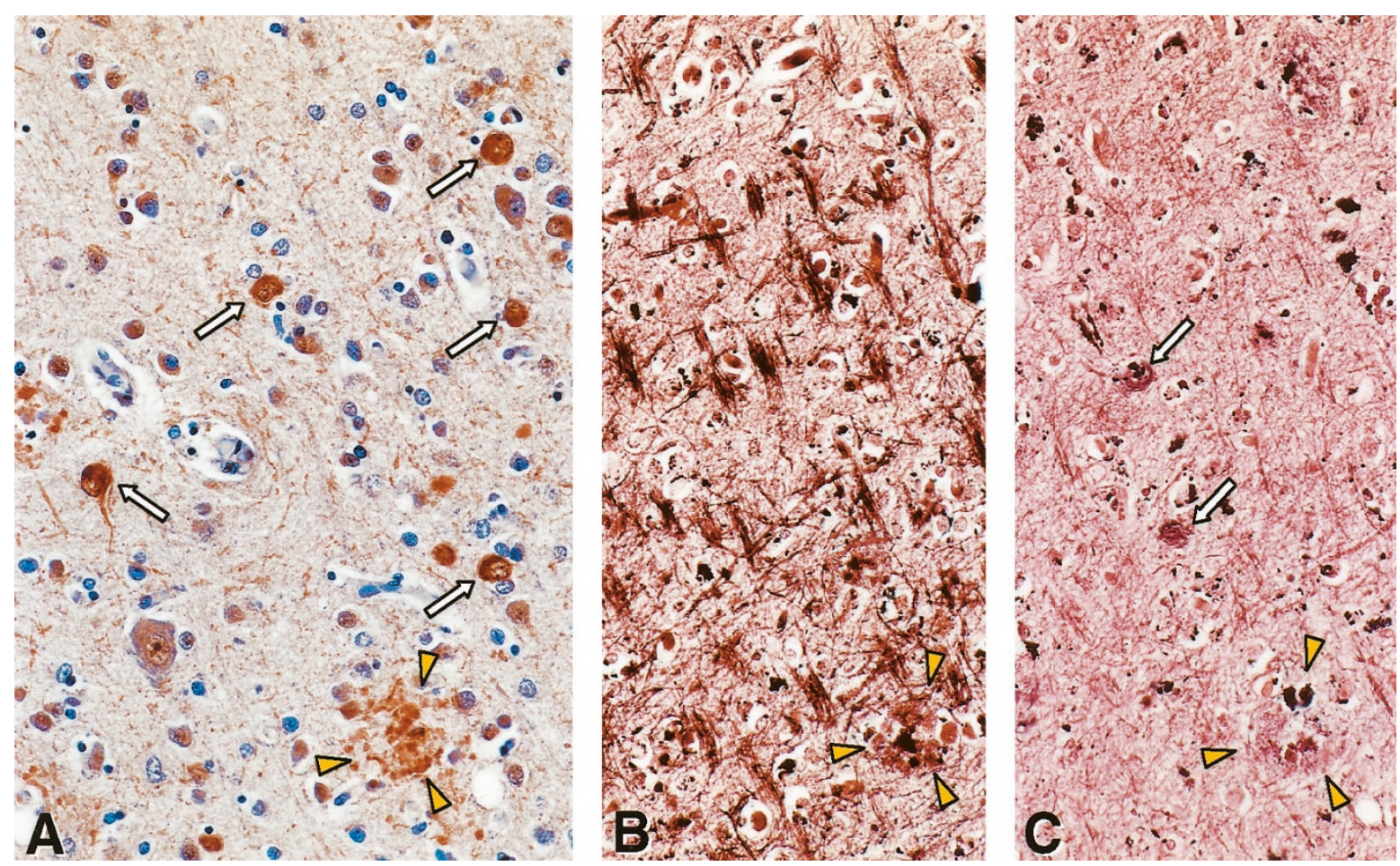

FIGURE 3. Case 13 was classified as Stage IV by silver stain, but ubiquitin stains were interpreted as stage V-VI. Re-review of the silver stains revealed that dark staining of the crossing myelinated fibers characteristic of the visual cortex interfered with detection of tangles. A, ubiquitin immunostain highlights numerous tangles (arrows) in the primary visual cortex. A plaque is also present (arrowheads). B, silver impregnation stain of the same region of the primary visual cortex. Only a plaque (arrowheads) is visible; tangles are obscured by crossing fibers (Microwave King stain). C, the visual association cortex has a cleaner silver-stained background than the primary visual cortex. Nevertheless, it is still more difficult to appreciate tangles (arrows) or plaques (arrowheads) compared with the ubiquitin stain in A (Microwave King stain). (All three panels from Case 13, original magnification, $80 \times$.)

TABLE 1. Consortium to Establish a Registry for Alzheimer's Disease Diagnoses Based on Ubiquitin Immunostains

\begin{tabular}{|c|c|c|c|c|c|c|c|c|c|}
\hline $\begin{array}{c}\text { Case } \\
\text { No. }\end{array}$ & Age & Demented? & Silver & Rev \#1a & Rev \#1b & Rev \#2a & Rev \#2b & Rev \#3 & Rev \#4 \\
\hline 1 & 56 & no & non-AD & non-AD & non-AD & non-AD & non-AD & possible & non-AD \\
\hline 2 & 93 & no & possible & non-AD & non-AD & non-AD & non-AD & non-AD & non-AD \\
\hline 3 & 76 & no & possible & possible & non-AD & possible & possible & possible & non-AD \\
\hline 4 & 86 & no & possible & possible & non-AD & non-AD & non-AD & non-AD & possible \\
\hline 5 & 92 & no & possible & possible & possible & possible & possible & possible & possible \\
\hline 6 & 76 & yes & possible & non-AD & probable & possible & possible & possible & possible \\
\hline 7 & 83 & yes & probable & definite & possible & probable & probable & poss-prob & definite \\
\hline 8 & 82 & yes & probable & definite & probable & possible & possible & possible & probable \\
\hline 9 & 78 & yes & probable & definite & definite & probable & probable & definite & probable \\
\hline 10 & 74 & yes & definite & definite & definite & definite & definite & definite & definite \\
\hline 11 & 76 & yes & definite & definite & definite & definite & definite & definite & definite \\
\hline 12 & 89 & yes & definite & definite & definite & definite & probable & probable & definite \\
\hline 13 & 76 & yes & definite & definite & definite & definite & definite & definite & definite \\
\hline 14 & 80 & yes & definite & definite & definite & definite & definite & probable & definite \\
\hline 15 & 77 & yes & definite & definite & definite & definite & definite & definite & definite \\
\hline 16 & 74 & yes & definite & definite & definite & definite & definite & definite & definite \\
\hline
\end{tabular}

$\mathrm{AD}$, Alzheimer's disease.

The official Alzheimer Disease Research Center diagnoses based on silver impregnation stains are shown for comparison (bold type).

Reviewers 1 and 2 each scored the immunostains a second time after intervals of 4 to 8 months.

substitute the major components of this protocol with another primary, secondary, or conjugated horseradish peroxidase, however, the signal may vary. Thus, ubiquitin immunostains may be substituted for labor-intensive and often fickle silver impregnation stains in applying CERAD recommendations for community-based diagnosis of Alzheimer's disease (7).
Although ubiquitin stains were highly accurate and reproducible for evaluating brains by CERAD diagnostic criteria, difficulties with distinguishing globose tangles from Lewy bodies were noted by one reviewer. A relatively new commercially available $\alpha$-synuclein immunostain for Lewy pathology may prove helpful in this regard. Some of the variability in Braak staging is attributable to the fact 
TABLE 2. Braak Staging of Cases Based on Ubiquitin Immunostains

\begin{tabular}{|c|c|c|c|c|c|c|c|c|c|}
\hline $\begin{array}{c}\text { Case } \\
\text { No. }\end{array}$ & Demented? & Silver & $\begin{array}{l}\text { Rev } \\
\# 1 \mathrm{a}\end{array}$ & Rev \#1b & $\begin{array}{l}\text { Rev } \\
\text { \#2a }\end{array}$ & $\begin{array}{l}\text { Rev } \\
\# 2 b\end{array}$ & $\begin{array}{l}\text { Rev } \\
\# 3 a\end{array}$ & $\begin{array}{l}\text { Rev } \\
\# 3 b\end{array}$ & $\begin{array}{c}\text { Rev } \\
\# 4\end{array}$ \\
\hline 1 & no & I & I & I & I & I & III & II & I \\
\hline 2 & no & I & I & I & I & I & $\mathrm{V}$ & II & I \\
\hline 3 & no & II & II-III & II & II-III & I & III & II-III & I \\
\hline 4 & no & III & III & I & III & I & III & III & I \\
\hline 5 & no & III & III & III & III & III & IV & IV & III \\
\hline 6 & yes & III & I & II & III & II-III & $\mathrm{V}$ & III & I \\
\hline 7 & $5 \mathrm{y}$ & II & III & II & III & II & $\mathrm{V}$ & II & I \\
\hline 8 & yes & IV & IV & III & IV & IV & III-IV & IV & $\mathrm{V}$ \\
\hline 9 & $5 \mathrm{y}$ & $\mathbf{V}$ & III-IV & III & $\mathrm{V}$ & III & $\mathrm{V}-\mathrm{VI}$ & V & III \\
\hline 10 & yes & III & III & III & III & III & III & III & I-II \\
\hline 11 & $4 \mathrm{y}$ & IV & III & IV & IV & IV & III-IV & IV & IV \\
\hline 12 & $3 y$ & IV & IV-V & IV & IV & II-III & $\mathrm{V}$ & IV & III \\
\hline 13 & yes & IV & $\mathrm{V}$ & $\mathrm{V}$ & VI & VI & $\mathrm{V}$ & $\mathrm{V}$ & VI \\
\hline 14 & $13 \mathrm{y}$ & V & IV & IV & IV & IV & IV-V & V & III \\
\hline 15 & $10 \mathrm{y}$ & VI & VI & $\mathrm{V}$ & V-VI & V & VI & VI & $\mathrm{V}$ \\
\hline 16 & $20 \mathrm{y}$ & VI & VI & VI & VI & VI & VI & VI & $\mathrm{V}$ \\
\hline
\end{tabular}

The Braak stage derived from silver impregnation stains is shown in bold print for comparison.
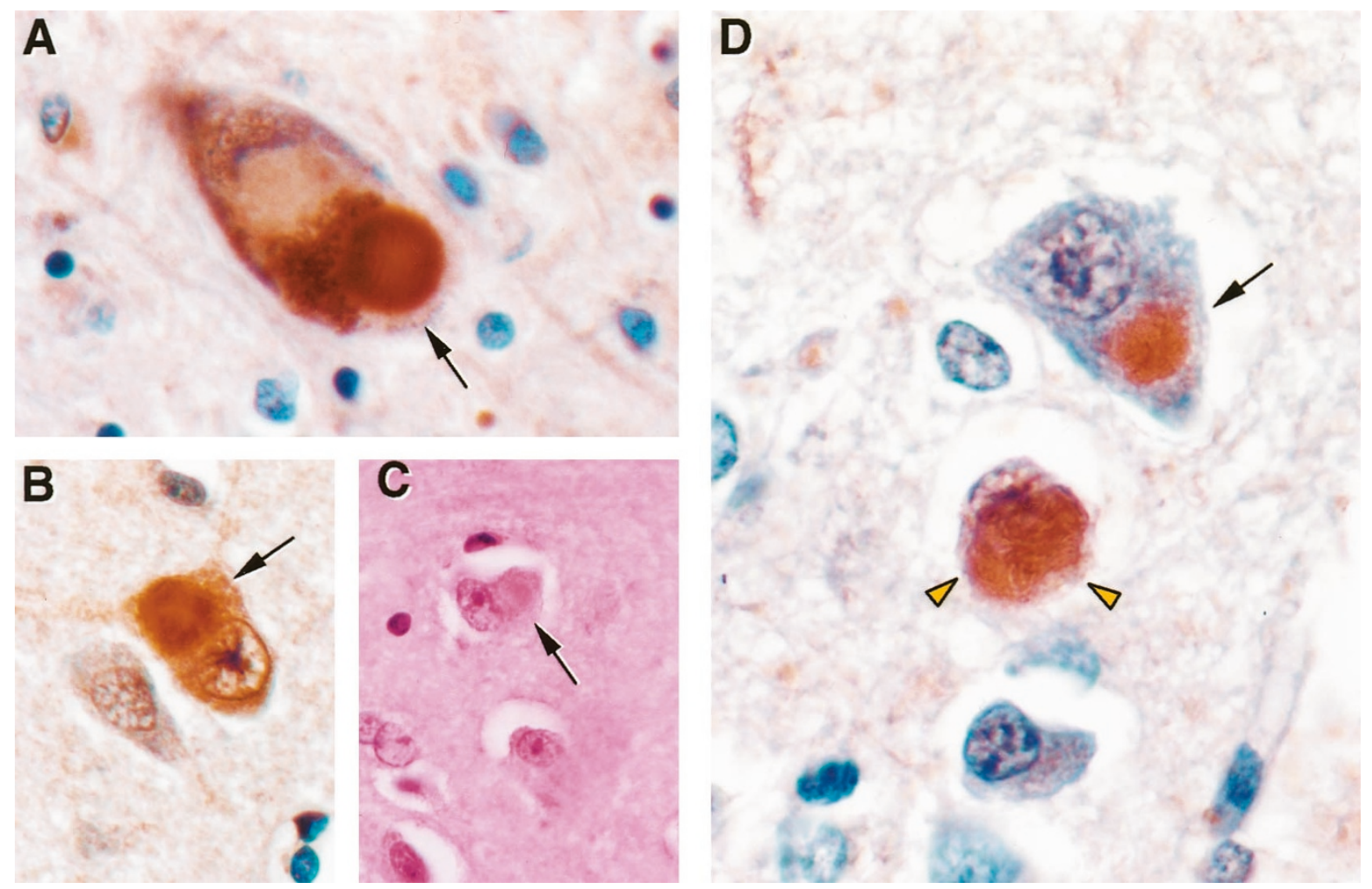

FIGURE 4. Staining of Lewy bodies. A, typical appearance of a mature Lewy body (arrow) in a pigmented neuron of the substantia nigra. The diaminobenzidine product is readily distinguishable from the darker endogenous pigment (Case 10, 160×, ubiquitin). B, Lewy bodies (arrow) typically are more indistinct in cortical neurons but are still readily identified on ubiquitin immunostains (Case 7 , original magnification, $160 \times$ ). C, re-review of the corresponding hematoxylin and eosin of Case 7 revealed cortical Lewy bodies (arrow) that were previously missed (original magnification, $160 \times$ ). D, making a distinction between globose tangles (arrowheads)—fibrillar accumulations that fill the cytoplasm of small, round neurons - and Lewy bodies (arrow) - which form discrete, rounded homogeneous intracytoplasmic inclusions-is critical for accurate evaluation of Alzheimer's disease stage or concurrent Parkinson's disease pathology (Case 11, original magnification, 300×, ubiquitin).

that the staging protocol itself is more difficult, relying on correct cytoarchitectural identification of specific brain regions rather than simply scoring the level of maximum involvement in any neocortical region. Because of the many silver-positive normal structures in the brain (Fig. 3), it is likely that there would be even greater variability in Braak staging based on silver impregnation stains. Thus, ubiquitin immunostains should prove useful for Braak staging as well, which is performed mainly in the academic setting.

It is important to realize that the level of diagnostic accuracy in this study was achieved by examining ubiquitin immunostains in isolation. The addition of $\mathrm{H} \& \mathrm{E}$ and amyloid stains would greatly enhance the evaluation of vascular contributions to 
dementia. The use of $\alpha$-synuclein immunostains to delineate Lewy bodies and Lewy neurites, tau immunostains to highlight tangles, and $\beta$-amyloid immunostains to identify vascular and cerebral amyloid deposits can also supplement information gathered from $\mathrm{H} \& \mathrm{E}$, silver, Congo red, and ubiquitin stains. Ubiquitin immunochemistry on its own, however, allowed pathologists-in-training who had brief exposure to neuropathology to render diagnoses highly comparable to those made by an experienced neuropathologist.

Thus, for the practicing community pathologist, the addition of a single immunostain for ubiquitin comprises a simple, effective diagnostic aid for evaluating Alzheimer's and Parkinson's/Lewy body neuropathology. Ubiquitin immunohistochemistry in combination with routine H\&E stains should prove accurate and reproducible for distinguishing Alzheimer's disease from vascular or Lewy body dementias.

Acknowledgments: We thank Susan Embry of Duke Photopathology for her assistance with the digitized composite images. This work was supported by $\mathrm{Na}$ tional Institute on Aging grant AG05128 and GlaxoWellcome.

\section{REFERENCES}

1. Cummings J, Vinters H, Cole G, Khachaturian Z. Alzheimer's disease: etiologies, pathophysiology, cognitive reserve and treatment opportunities. Neurol 1998;51(Suppl 1):S2-17.

2. Price DL, Sisodia SS, Borchelt DR. Genetic neurodegenerative diseases: the human illness and transgenic models. Science 1998;282:1079-83.

3. Chu CT, Pizzo SV. $\alpha_{2}$-Macroglobulin, complement, and biologic defense: antigens, growth factors, microbial proteases, and receptor ligation. Lab Invest 1994;71:792-812.

4. Saunders AM, Strittmatter W, Schmechel D, St. GeorgeHyslop P, Pericak-Vance M, Joo S, et al. Association of apolipoprotein E allele $\epsilon 4$ with late-onset familial and sporadic
Alzheimer's disease. Neurology 1993;43:1467-72.

5. Blacker D, Wilcox MA, Laird NM, Rodes L, Horvath SM, Go $\mathrm{RC}$, et al. Alpha-2 macroglobulin is genetically associated with Alzheimer disease. Nat Genet 1998;19:357-60.

6. Saunders AM, Hulette CM, Welsh KA, Schmechel DE, Crain B, Burke JR, et al. Specificity, sensitivity and predictive value of apolipoprotein E genotyping in a consecutive autopsy series of sporadic Alzheimer disease patients. Lancet 1996; 348:90-3.

7. Mirra SS, Hart MN, Terry RD. Making the diagnosis of Alzheimer's disease: a primer for practicing pathologists. Arch Pathol Lab Med 1993;117:132-44.

8. Mirra SS, Gearing M, McKeel DW, Crain BJ, Hughes JP, Van Belle G, et al. Interlaboratory comparison of neuropathology assessments in Alzheimer's disease: a study of the Consortium to Establish a Registry for Alzheimer's Disease (CERAD). J Neuropathol Exp Neurol 1994;53:303-15.

9. Braak H, Braak E. Neuropathological stageing of Alzheimerrelated changes. Acta Neuropathol 1991;82:239-59.

10. National Institute on Aging and Reagan Institute Working Group on Diagnostic Criteria for the Neuropathological Assessment of Alzheimer's Disease. Consensus recommendations for the postmortem diagnosis of Alzheimer's disease. Neurobiol Aging 1997;18(Suppl 4):51-2.

11. Hershki A, Ciechanover A. The ubiquitin system. Ann Rev Biochem 1998;67:425-79.

12. Hulette C, Mirra S, Wilkinson W, Heyman A, Fillenbaum G, Clark C. A prospective clinical-neuropathological study of Parkinson's features in Alzheimer's disease: the CERAD experience, Part IX. Neurol 1995;45:1991-5.

13. Gearing M, Mirra SS, Hedreen JC, Sumi SM, Hansen LA, Heyman A. The consortium to establish a registry for Alzheimer's disease (CERAD). Part X. Neuropathology confirmation of the clinical diagnosis of Alzheimer's disease. Neurology 1995;45:461-6.

14. Hulette CM, Welsh-Bohmer KA, Crain B, Szymanski MH, Sinclaire NO, Roses AD. The Joseph and Kathleen Bryan Alzheimer's Disease Research Center Experience. Arch Pathol Lab Med 1997;121:615-8.

15. Lloyd B, Brinn N, Burger PC. Silver-staining of senile plaques and neurofibrillary change in paraffin-embedded tissues. J Histotechnol 1985;8:155-6.

16. McKeith IG, Galasko D, Kosaka K, Perry EK, Dickson DW, Hansen LA, et al. Consensus guidelines for the clinical and pathologic diagnosis of dementia with Lewy bodies (DLB): report of the consortium on DLB international workshop. Neurology 1996;47:1113-24. 